\title{
The Current Strategies for Neoadjuvant Chemotherapy in Muscle Invasive Bladder Cancer: The Place of Immunotherapy in Future
}

\author{
๑ Özlem Ercelep \\ Marmara University Pendik Training and Research Hospital, Clinic of Medical Oncology, Istanbul, Turkey
}

\begin{abstract}
Muscle invasion is present in approximately thirty percent of bladder cancers. Radical cystectomy and bilateral pelvic lymph node dissection following neoadjuvant chemotherapy is the recommended treatment for muscle invasive bladder cancer. Local and distant relapses are seen in a significant proportion of patients with surgery alone. Micrometastases at the time of diagnosis that cannot be detected by imaging may be responsible for these relapses. The aim of neoadjuvant and adjuvant therapies is to eliminate these micrometastases. Several randomized trials have shown that platinum-based combination neoadjuvant chemotherapy can improve survival outcomes, compared with locoregional treatment alone. Although the proportion of patients receiving treatment has increased compared to previous years, it is still low. Immunotherapies in neoadjuvant treatment are promising in the appropriate patient group. In this article, we aimed to review current neoadjuvant treatment strategies in muscle invasive bladder cancer in the literature.
\end{abstract}

Keywords: Bladder cancer, chemotherapy, neoadjuvant therapy

\section{Introduction}

Although the gold standard treatment in bladder cancer is surgical, the rate of cure varies between $50-65 \%$ by surgery alone (1). It is seen that systemic treatment applications are of great importance considering the low chance of obtaining a cure and low survival rates in patients treated with surgery alone in case of the tumor invades the muscle layer. Urothelial (transitional cell) carcinoma accounts for about ninety percent of bladder cancers in Europe and North America. In other parts of the world, non-urothelial bladder cancers are more common. Although neoadjuvant cisplatin-based chemotherapies provide a survival benefit compared to local treatments alone, only twenty percent of patients receive neoadjuvant chemotherapy before radical cystectomy $(2,3,4,5)$. The reasons such as physicians not believing in the benefit of the treatment, fear of the side effects of the treatment, not guiding the patients for chemotherapy due to the concerns of delay in curative treatment, and impaired renal function and poor performance prevent patients from receiving treatment.

All patients should be clinically staged prior to treatment in bladder cancer. It is necessary to start treatment as soon as possible after the diagnosis and staging. In an observational study, it was shown that delay of 8 weeks or more in neoadjuvant therapy was associated with the increase in stage in 2,200 patients (6).

The survival benefit of cisplatin-based chemotherapies before surgery has been demonstrated in the meta-analyses of 11 randomized studies (7). Compared with local therapy alone, neoadjuvant cisplatin-based treatments improve 5-year survival from $45 \%$ to $50 \%$, while improve disease-free survival by $9 \%$. However, there are no randomized studies comparing neoadjuvant chemotherapy regimens. A clear difference between regimens was not shown in retrospective studies $(8,9)$. In the largest one of those retrospective studies, the data of 935 patients with node negative (N0) muscle invasive bladder cancer (T2-T4a), from 19 centers from North America and Europe were analyzed. In that study, $64 \%$ of patients received cisplatin gemcitabine, 20\% methotrexate, vinblastine, doxorubicin, and cisplatin (MVAC) and $15 \%$ other regimens. There was no difference between the chemotherapy regimens in terms of pathological complete response rates (9).

In the intergroup-0080 trial, surgery alone was compared with neoadjuvant classical MVAC regimen (every 3 days for 28 days) followed by surgery in 307 patients with T2-4N0M0 disease. In the study with a median follow-up time of 8.7 years, median survival in the neoadjuvant therapy arm was 77 months,

Cite this article as: Ercelep Ö. The Current Strategies for Neoadjuvant Chemotherapy in Muscle Invasive Bladder Cancer: The Place of Immunotherapy in Future. Bull Urooncol 2020;19(3):104-106

Address for Correspondence: Özlem Ercelep, Marmara University Pendik Training and Research Hospital, Clinic of Medical Oncology, İstanbul, Turkey Phone: +90 2166570606 E-mail: ozlembalvan@yahoo.com ORCID-ID: orcid.org/0000-0001-5892-3519 
whereas it was 46 months in the surgery alone arm $(p=0.06)$. While the 5-year overall survival benefit in the study was 5\%, it was seen that this benefit was more pronounced in T3-4 tumors with $20 \%$. In this study, the 5-year survival rate of patients with complete pathological response (pT0) was $85 \%$. In the study, the pathological complete response (15\% versus 38\%, $\mathrm{p}<0.001$ ) was found better in the arm receiving neoadjuvant chemotherapy compared to surgery alone. In summary, in that study, patients who received neoadjuvant therapy had a higher pathological complete response rate, and this was associated with increased survival (10).

The dose-dense MVAC treatment used in conjunction with the granulocyte colony stimulating factor every two weeks showed superiority in survival compared to classical MVAC treatment in locally advanced and metastatic disease $(11,12)$. After these studies, single arm, two-phase 2 studies were conducted to investigate the neoadjuvant use of dose-dense MVAC (filgrastim every 14 days, 3 or 4 cycles depending on toxicity). In the first study, the pathological full response rate was $26 \%$ (43\% of the patients were clinically node positive patients in this study), whereas in the other study, the pathological full response rate was $38 \%$ (in this study, $7 \%$ of the patients were clinically node positive patients) $(13,14)$. The dose-dense MVAC treatment regimen is considered an alternative treatment regimen, especially in patients who have short duration until surgery and are clinically NO patients.

Compared with MVAC treatment, gemcitabine cisplatin (GC) treatment in metastatic patients showed similar efficacy and less toxicity (15). There are no randomized studies in neoadjuvant use of this regimen. In a meta-analysis conducted in 2016, the data of 13 retrospective studies were analyzed. Although this meta-analysis showed similar response rates between GC/ carboplatin treatment and MVAC treatment, survival results were found inferior in gemcitabine-platinum arm. In the analysis made by excluding carboplatin data, GC treatment was found inferior (16).

The effectiveness of neoadjuvant cisplatin, methotrexate, vinblastin therapy (methotrexate, vinblastin, cisplatin, leukoverine, 3 cycles every 28 days) was compared with local treatment in 976 high-grade patients in a phase 3 trial. In this study, $6 \%$ absolute survival benefit was shown at the end of 10 years of follow-up (36\% vs 30\%) (17).

In the Nordic Cystectomy 1 trial, 311 patients were enrolled in the T1-4N0M0 phase; one group underwent a combination regimen with neoadjuvant cisplatin (cisplatin and adriamycin) followed by radiotherapy and surgery, and the other group underwent surgery after radiotherapy without neoadjuvant therapy (18). While the 5-year overall survival benefit in the study was shown in T1-2 tumors, a 15\% difference was found in favor of neaoadjuvant therapy in patients with T3-4 stage $(p=0.03)$. The same study group performed Nordic cystectomy 2 trial and examined 317 patients by dividing them into neoadjuvant therapy (cisplatin and methotrexate) and surgery alone arm (19). There was no difference between the two arms in terms of survival. When Nordic 1 and 2 trials were analyzed together, it was observed that 5-year survival increased from $48 \%$ to $56 \%$ with neoadjuvant therapy $(p=0.049)$.
In one of the studies conducted to investigate the effect of response obtained with neoadjuvant therapy on survival, the results of 449 patients with T2-4NxM0 tumor who were included in prospective Nordic cystectomy trials and had a pathological complete response with neoadjuvant therapy were retrospectively analyzed. The difference in 5 year survival between the group with and without pathological complete response was shown (57.1\% versus $88.2 \%)(p=0.001)(20)$. As a result of the study, it was demonstrated that a significant advantage in 5-year survival in patients with complete pathological response with neoadjuvant therapy was gained.

\section{Treatment in Patients with Kidney Failure}

Urinary system obstruction should be investigated primarily in patients with bladder cancer presenting with kidney failure. If there is obstruction and if the kidney functions are normalized by placing a stent in the ureter or percutaneous nephrostomy, a standard dose of cisplatin can be given. If kidney failure is not caused by obstruction and if stent placement or nephrostomy does not improve kidney failure, carboplatin can be used instead of cisplatin (21). Data show that carboplatin is less effective than cisplatin in urothelial cancers $(22,23,24,25)$. However, there are no randomized studies comparing cisplatin and carboplatin as neoadjuvant therapy.

\section{Neoadjuvant Immunotherapy}

With the demonstration of the effectiveness of immunotherapies in metastatic urothelial cancers, the use of immunotherapies in neoadjuvant therapy has also been investigated. In two different phase 2 studies, the place of atezolizumab and pembrolizumab in neoadjuvant therapy was investigated. In the PURE-01 study, the effectiveness of neoadjuvant pembrolizumab therapy was evaluated in 50 patients with muscle invasive bladder cancer. In this study, pathological complete response as the primary outcome was $42 \%$ and reduction in stage was $54 \%$. In subgroup analysis, benefit was seen in patients with pdl1 $\geq 10 \%$ or with high tumor mutation. In the second study, the effectiveness of atezolizumab in neoadjuvant therapy was evaluated and similar results were obtained with pemborolizumab $(26,27)$. If these results are confirmed by randomized phase 3 studies, immunotherapy in neoadjuvant therapy may be used in the near future.

In the light of this information, neoadjuvant therapy is recommended in clinical guidelines beginning from the T2N0M0 stage or in the disease with nodal involvement (TxN1$3 \mathrm{M0}$ ). The contribution of neoadjuvant therapy to survival does not appear to be very high, since randomized studies and studies in meta-analysis included patients in the T2 stage. While MVAC systemic therapy is preferred in healthy individuals under the age of 70, cisplatin-gemcitabine therapy is the preferred chemotherapy regimen for individuals over 70 years of age or with comorbidity. However, in practice, cisplatin-gemcitabine therapy is applied in most centers for reasons such as treatment toxicity and difficulty of administration.

\section{Acknowledgements}

Publication: The results of the study were not published in full or in part in form of abstracts. 
Contribution: There is not any contributors who may not be listed as author.

Financial Disclosure: The author declared that this study received no financial support.

\section{Ethics}

Peer-review: Internally peer-reviewed.

\section{References}

1. Raghavan D, Shipley WU, Garnick MB, et al. Biology and Management of Bladder Cancer. N Engl J Med 1990;332:1129-1138.

2. Miles BJ, Fairey AS, Eliasziw M, et al. Referral and treatment rates of neoadjuvant chemotherapy in muscle-invasive bladder cancer before and after publication of a clinical practice guideline. Can Urol Assoc J 2010;4:263-267.

3. Porter MP, Kerrigan MC, Donato BM, Ramsey SD. Patterns of use of systemic chemotherapy for Medicare beneficiaries with urothelial bladder cancer. Urol Oncol 2011;29:252-258.

4. Apolo AB, Kim JW, Bochner BH, et al. Examining the management of muscle-invasive bladder cancer by medical oncologists in the United States. Urol Oncol 2014;32:637-644.

5. Raj GV, Karavadia S, Schlomer B, et al. Contemporary use of perioperative cisplatin-based chemotherapy in patients with muscleinvasive bladder cancer. Cancer 2011;117:276-282.

6. Audenet F, Sfakianos JP, Waingankar N, et al. A delay $\geq 8$ weeks to neoadjuvant chemotherapy before radical cystectomy increases the risk of upstaging. Urol Oncol 2019;37:116-122.

7. Advanced Bladder Cancer Meta-analysis Collaboration. Neoadjuvant chemotherapy in invasive bladder cancer: a systematic review and meta-analysis. Lancet 2003;361:1927-1934.

8. Galsky MD, Pal SK, Chowdhury $\mathrm{S}$, et al. Comparative effectiveness of gemcitabine plus cisplatin versus methotrexate, vinblastine, doxorubicin, plus cisplatin as neoadjuvant therapy for muscleinvasive bladder cancer. Cancer 2015;121:2586-2593.

9. Zargar H, Espiritu PN, Fairey AS, et al. Multicenter assessment of neoadjuvant chemotherapy for muscle-invasive bladder cancer. Eur Urol 2015;67:241-249.

10. Grossman HB, Natale RB, Tangen CM, et al. Neoadjuvant chemotherapy plus cystectomy compared with cystectomy alone for locally advanced bladder cancer. N Engl J Med 2003;349:859-866.

11. Sternberg CN, de Mulder P, Schornagel JH, et al. Seven-year update of an EORTC phase III trial of high-dose intensity M-VAC chemotherapy and G-CSF versus classic M-VAC in advanced urothelial tract tumours. Eur J Cancer 2006;42:50-54.

12. Sternberg $\mathrm{CN}$, de Mulder $\mathrm{PH}$, Schornagel $\mathrm{JH}$, et al. Randomized phase III trial of high-dose-intensity methotrexate, vinblastine, doxorubicin, and cisplatin (MVAC) chemotherapy and recombinant human granulocyte colony-stimulating factor versus classic MVAC in advanced urothelial tract tumors: European Organization for Research and Treatment of Cancer Protocol no. 30924. I Clin Oncol 2001;19:2638-2646.
13. Choueiri TK, Jacobus S, Bellmunt J, et al. Neoadjuvant dosedense methotrexate, vinblastine, doxorubicin, and cisplatin with pegfilgrastim support in muscle-invasive urothelial cancer: pathologic, radiologic, and biomarker correlates. J Clin Oncol 2014;32:1889-1894.

14. Plimack ER, Hoffman-Censits JH, Viterbo $\mathrm{R}$, et al. Accelerated methotrexate, vinblastine, doxorubicin, and cisplatin is safe, effective, and efficient neoadjuvant treatment for muscle-invasive bladder cancer: results of a multicenter phase II study with molecular correlates of response and toxicity. J Clin Oncol 2014;32:1895-1901.

15. von der Maase $H$, Hansen SW, Roberts JT, et al. Gemcitabine and cisplatin versus methotrexate, vinblastine, doxorubicin, and cisplatin in advanced or metastatic bladder cancer: results of a large, randomized, multinational, multicenter, phase III study. J Clin Oncol 2000;19:3068-3077.

16. Yin M, Joshi M, Meijer RP, et al. Neoadjuvant Chemotherapy for Muscle-invasive Bladder Cancer: A Systematic Review and Two-Step Meta-Analysis The Oncologist 2016;21:708-715.

17. Neoadjuvant cisplatin, methotrexate, and vinblastine chemotherapy for muscle-invasive bladder cancer: a randomised controlled trial. International collaboration of trialists. Lancet 1999;354:533-540.

18. Malmström PU, Rintala E, Wahlqvist R, et al. Five-year followup of a prospective trial of radical cystectomy and neoadjuvant chemotherapy: Nordic Cystectomy Trial I. The Nordic Cooperative Bladder Cancer Study Group. J Urol 1996;155:1903-1906.

19. Sherif A, Rintala E, Mestad O, et al. Neoadjuvant CisplatinMethotrexate Chemotherapy for Invasive Bladder Cancer- Nordic Cystectomy Trial 2. Scand J Urol Nephrol 2002;36:419-425.

20. Rosenblatt R, Sherif A, Rintala E, et al. Pathologic Downstaging Is a Surrogate Marker for Efficacy and Increased Survival Following Neoadjuvant Chemotherapy and Radical Cystectomy for MuscleInvasive Urothelial Bladder Cancer. Eur Urol 2012;61:1229-1238.

21. Mertens LS, Meijer RP, Kerst JM, et al. Carboplatin based induction chemotherapy for nonorgan confined bladder cancer--a reasonable alternative for cisplatin unfit patients? J Urol 2012;188:1108-1113.

22. Trump DL, Elson P, Madajewicz S, et al. Randomized phase II evaluation of carboplatin and CHIP in advanced transitional cell carcinoma of the urothelium. The Eastern Cooperative Oncology Group. J Urol 1990;144:1119-1122.

23. Raghavan D. Progress in the chemotherapy of metastatic cancer of the urinary tract. Cancer 2003;97:2050-2055.

24. Raabe NK, Fossa SD, Parø G. Phase II study of carboplatin in locally advanced and metastatic transitional cell carcinoma of the urinary bladder. Br J Urol 1989;64:604-607.

25. Hansen PV, Glavind K, Panduro J, Pedersen M. Paternity in patients with testicular germ cell cancer: pretreatment and post-treatment findings. Eur J Cancer 1991;27:1385-1389.

26. Necchi A, Anichini A, Raggi D, et al. Pembrolizumab as Neoadjuvant Therapy Before Radical Cystectomy in Patients with Muscle-Invasive Urothelial Bladder Carcinoma (PURE-01): An Open-Label, SingleArm, Phase II Study. J Clin Oncol 2018:36:3353-3360.

27. Powles T, Rodriguez-Vida A, Duran I, et al. A phase II study investigating the safety and efficacy of neoadjuvant atezolizumab in muscle invasive bladder cancer (ABACUS). J Clin Oncol 2018;36:4506. 